\title{
C-mannosylation of human hyaluronidase 1: Possible roles for secretion and enzymatic activity
}

\author{
YUKI GOTO $^{1}$, YUKI NIWA ${ }^{1}$, TAKEHIRO SUZUKI ${ }^{2}$, NAOSHI DOHMAE ${ }^{2}$, KAZUO UMEZAWA $^{3}$ and SIRO SIMIZU ${ }^{1}$ \\ ${ }^{1}$ Department of Applied Chemistry, Faculty of Science and Technology, Keio University, Yokohama; \\ ${ }^{2}$ Global Research Cluster, RIKEN; ${ }^{3}$ Department of Molecular Target Medicine Screening, \\ Aichi Medical University School of Medicine, Japan
}

Received February 10, 2014; Accepted April 16, 2014

DOI: $10.3892 /$ ijo.2014.2438

\begin{abstract}
Protein glycosylation, one of the post-translational modifications, is important for many protein functions, such as protein stability, folding and secretion. In the protein glycosylation, $C$-mannosylation was first identified in ribonuclease 2 , and some proteins have been reported to be $C$-mannosylated; however, effects of its modifications for target proteins remain unclear. Hyaluronidase 1 (HYAL1), degrading hyaluronic acid (HA), has two predicted $C$-mannosylation sites at $\operatorname{Trp}^{130}$ and $\operatorname{Trp}^{321}$. In this study, we examined whether HYAL1 is $C$-mannosylated or not, and the effect of $C$-mannosylation on HYAL1. Using mass spectrometry, we first demonstrated that intracellular HYAL1 is $C$-mannosylated at $\operatorname{Trp}^{130}$ but not at $\operatorname{Tr}^{321}$. Surprisingly, although HYAL1 was secreted into conditioned medium and it possessed enzymatic activity, secreted HYAL1 was not $C$-mannosylated. Computer simulation demonstrated that $C$-mannosylation of HYAL1 at $\operatorname{Trp}^{130}$ changed conformation of the catalytic active site, and faced $\mathrm{Glu}^{131}$ in the opposite direction toward its substrate, HA, indicating that $C$-mannosylation will negatively regulate its secretion, and will attenuate its enzymatic activity. Taken together, this is the first report that demonstrates the presence of $C$-mannosylation among HYAL family proteins, and our results suggest possible roles of $C$-mannosylation for secretion and enzymatic activity.
\end{abstract}

\section{Introduction}

$C$-mannosylation is a unique type of glycosylation in which $\alpha$-D-mannose is directly attached to the indole $\mathrm{C}_{2}$ carbon atom of a tryptophan residue via a C-C linkage $(1,2)$. It is observed within the sequence motif Trp-Xaa-Xaa-Trp/ Cys (Xaa represents any amino acids) in proteins, and the N-terminus tryptophan may be $C$-mannosylated $(3,4)$. Some proteins have been reported to be $C$-mannosylated, for example, in ribonuclease 2 (5), F-spondin (6), throm-

Correspondence to: Professor Siro Simizu, Keio University, 3-14-1 Hiyoshi, Kohoku-ku, Yokohama 223-8522, Japan

E-mail: simizu@applc.keio.ac.jp

Key words: glycosylation, mass spectrometry, C-mannosylation, hyaluronidase bospondin (7), a disintegrin and metalloproteinase with thrombospondin motif (ADAMTS)-like 1/punctin-1 (8) and interleukin-21 receptor (9); however, the biological functions of $C$-mannosylation remain largely unknown. Recently, the responsible $C$-mannosyltransferase for thrombospondin type-1 repeats (TSR-1), dpy19, was identified in $C$. elegans (10). $C$-mannosylation is expected to affect protein polarity because the polar mannose is attached to the non-polar tryptophan. Moreover, the attachment of mannose to tryptophan is expected to induce a conformational change of the target proteins. Therefore, $C$-mannosylation might affect protein functions, such as protein stability, secretion, intracellular localization and even enzymatic activity. In fact, it was reported that $C$-mannosylation interferes with the secretion of ADAMTS-like 1/punctin-1 (8). Moreover, it is also known that $C$-mannosylated peptides, derived from TSR-1, enhance lipopolysaccharide-induced signaling, such as tumor necrosis factor alpha and c-jun N-terminal kinase in RAW264.7 cells $(11,12)$. However, little is known about other functions of $C$-mannosylation, so further detailed investigations are required to clarify the roles of its modification.

Hyaluronic acid (HA) is a component of extracellular matrices (ECMs). Hyaluronidases (HYALs) hydrolyze the $\beta 1-4$ linkage between $N$-acetylglucosamine and glucuronic acid of HA polymers. ECM degradation enzymes, such as matrix metalloproteinases and heparanase, have been known to promote cancer metastasis and invasion (13-15). In the same way, upregulation of HYALs, especially HYAL1, has been reported to correlate with tumor cell proliferation, migration, invasion and angiogenesis in various cancers, including breast, prostate and ovarian cancers (16-18). Moreover, HYAL1 is known to correlate with juvenile idiopathic arthritis (JIA). Defects of HYAL1 are not only the cause of mucopolysaccharidosis but are present in JIA (19).

In this investigation, we examined the presence of $C$-mannosylation in human HYAL1 and its role for HYAL1 functions. As a result, we show possible roles for secretion and enzymatic activity by $C$-mannosylation of HYAL1.

\section{Materials and methods}

Cell culture. Human fibrosarcoma HT1080 cells, purchased from Japanese Cancer Research Resources Bank (JCRB), were 
cultured in Dulbecco's modified Eagle's medium (DMEM; Nissui, Tokyo, Japan), supplemented with $10 \%$ (v/v) fetal bovine serum, $200 \mathrm{U} / \mathrm{ml}$ penicillin $\mathrm{G}, 200 \mathrm{mg} / 1$ kanamycin, $600 \mathrm{mg} / \mathrm{l} \mathrm{L}$-glutamine, and $2.25 \mathrm{~g} / 1 \mathrm{NaHCO}_{3}$ at $37^{\circ} \mathrm{C}$ in a humidified incubator with $5 \% \mathrm{CO}_{2}$.

Establishment of the HYAL1-overexpressing cell line. The human HYAL1-myc-his ${ }_{6}$ gene was amplified from a human prostate cancer LNCaP cell cDNA library and subcloned into the pCI-neo vector (Promega, Madison, WI). The permanent cell line expressing HYAL1-myc-his ${ }_{6}$ was established by transfecting the vector into HT1080 cells, followed by $400 \mu \mathrm{g} / \mathrm{ml} \mathrm{G} 418$ (Roche Applied Sciences, Indianapolis, IN) selection. The clonal cells that expressed high levels of myc-his ${ }_{6}$-tagged HYAL1 were designated HT1080-HYAL1-MH cells. The cells that were transfected with pCI-neo vector were designated HT1080-neo.

Western blot analysis. To perform western blot analysis, we used a slightly modified version of a previously described methods (20-23). Cells were lysed in a lysis buffer [50 mM Tris- $\mathrm{HCl}, \mathrm{pH} 7.5,150 \mathrm{mM} \mathrm{NaCl}, 0.1 \%$ sodium dodecyl sulfate (SDS), $1 \%$ Triton X-100, $1 \%$ sodium deoxycholate and $1 \mathrm{mM}$ phenylmethylsulfonyl fluoride] at $4^{\circ} \mathrm{C}$ with sonication. The lysates were centrifuged at 14,000 rpm for $10 \mathrm{~min}$, and the amount of protein was measured by staining with Coomassie Brilliant Blue (CBB) G-250 (Bio-Rad Laboratories, Hercules, CA). Loading buffer ( $350 \mathrm{mM}$ Tris-HCl, $\mathrm{pH} 6.8,30 \%$ glycerol, $0.012 \%$ bromophenol blue, 6\% SDS and 30\% 2-mercaptoethanol) was added to each lysate, which was subsequently boiled for $3 \mathrm{~min}$ and electrophoresed on SDS-polyacrylamide gels. Proteins were transferred to PVDF membranes and immunoblotted with anti-c-myc (Santa Cruz Biotechnology, Inc., Santa Cruz, CA) or anti- $\alpha$-tubulin (Sigma, St. Louis, MO) antibodies. Detection was performed with enhanced chemiluminescence reagent (Millipore Corporation, Billerica, MA).

Purification of recombinant protein from conditioned medium and whole-cell lysate. To purify recombinant HYAL1 from the conditioned medium, HT1080-HYAL1-MH cells were cultured in serum-free DMEM for $24 \mathrm{~h}$, and the conditioned media was concentrated on an ultrafiltration membrane and incubated with Ni-NTA agarose (Qiagen, Hilden, Germany) for $2 \mathrm{~h}$ at $4^{\circ} \mathrm{C}$. The Ni-NTA agarose was washed five times with phosphate-buffered saline (PBS) and eluted with $500 \mathrm{mM}$ imidazole.

To purify recombinant HYAL1 from the whole-cell lysate, HT1080-HYAL1-MH cells were lysed with binding buffer (50 mM Tris-HCl, pH 7.5, 0.5 M NaCl, $8 \mathrm{M}$ Urea, $20 \mathrm{mM}$ imidazole), and cell lysate was incubated with Ni-NTA agarose for $2 \mathrm{~h}$ at $4^{\circ} \mathrm{C}$. The Ni-NTA agarose was washed four times with PBS and eluted with $500 \mathrm{mM}$ imidazole.

The obtained samples were electrophoresed on SDS-polyacrylamide gels and stained with CBB R-250. The purified proteins were used for mass spectrometry $(24,25)$.

Mass spectrometry. Purified recombinant HYAL1 was subjected to SDS-polyacrylamide gels. After CBB staining, the bands were excised and treated with $0.05 \mu \mathrm{g}$ of sequencing-grade modified trypsin (Promega) at $37^{\circ} \mathrm{C}$ for $12 \mathrm{~h}$ in $0.1 \mathrm{M}$ Tris- $\mathrm{HCl}, \mathrm{pH}$ 8.0. The digests were desalted using Zip TipC18 $\mu$ (Millipore Corporation) and applied to matrix-assisted laser desorption ionization time of flight mass spectrometry (MALDI-TOF MS) on a Ultraflex TOF/TOF MS (Bruker Daltonics, Bremen, Germany) in reflector mode using $\alpha$-cyano-4-hydroxycinnamic acid as the matrix. The selected peaks were subjected to MS/MS analysis in LIFT mode.

Measurement of hyaluronidase activity. To measure HYAL1 activity by in-gel digestion assay, we carried out the experiment as previously reported $(26,27)$. Equal numbers $\left(2.0 \times 10^{6}\right.$ cells) of HT1080-neo and HT1080-HYAL1-MH cells were cultured in serum-free DMEM for $24 \mathrm{~h}$ and concentrated by using Ni-NTA agarose. Ni-NTA-bound proteins were eluted, and the samples were electrophoresed on an SDS-polyacrylamide gel containing rooster comb HA $\left(0.2 \mathrm{mg} / \mathrm{ml}\right.$; Sigma) at $4^{\circ} \mathrm{C}$. The gel was washed twice with SDS extraction buffer $(50 \mathrm{mM}$ Tris-HCl, pH 7.5, $0.1 \mathrm{M} \mathrm{NaCl}, 2.5 \%$ Triton $\mathrm{X}-100)$ for $1 \mathrm{~h}$ and incubated in assay buffer ( $50 \mathrm{mM}$ sodium formate, $\mathrm{pH} 4.0$, $150 \mathrm{mM} \mathrm{NaCl}$ ) at $37^{\circ} \mathrm{C}$ for $24 \mathrm{~h}$. After incubation, the gel was stained with $0.5 \%$ alcian blue (Sigma) containing 20\% ethanol and $10 \%$ acetic acid solution for $2 \mathrm{~h}$ and destained with $25 \%$ methanol and $7.5 \%$ acetic acid solution. HYAL1 activity can be observed as a transparent band in the blue pigment background.

Docking model of HYALI and HA by computer simulation. We examined the conformational changes of HYAL1 when it was $C$-mannosylated and unmannosylated by using the Molecular Operating Environment (MOE; Chemical Computing Group Inc., Montreal, Canada) per previously reported methods $(28,29)$. The Protein Data Bank sequences Apis melliflora HYAL-HA complex (PDB ID: 1FCV) and human HYAL1 (PDB ID: 2PE4) were loaded into MOE. Hydrogen atoms were added, and the protonation states were assigned using the Protonate 3D tool of MOE. Using AMBER12:EHT, one of the calculation methods of force field, energy was minimized. The 2PE4 sequence was aligned with $1 \mathrm{FCV}$, removing the Apis melliflora HYAL and HA structure. Energy minimization was applied to human HYAL1 and HA. A 3D structure of the refined model was used for the Site Finder module of MOE, which can identify possible ligand-binding sites. Protein docking was performed by alpha sphere and excluded volumebased ligand-receptor docking (ASE-Dock), which is based on ligand-receptor interaction energies, and the resulting score was calculated as $\mathrm{U}_{\text {DOCK }} \cdot \mathrm{U}_{\mathrm{DOCK}}$ is expressed by the sum of $\mathrm{U}_{\text {refine }}$, the entire free energy of ligand-receptor interaction, and $\mathrm{U}_{\text {strain }}$, the free energy of ligand stability. In the ASE-Dock module, ligand atoms have alpha spheres within $1 \AA$ A Utilizing this property, models are created, and ligand atoms from many conformations that are generated by superposition with these points can be evaluated and scored by maximum overlap with alpha spheres and minimum overlap with the receptor atoms. Protein docking between human HYAL1 and dummy ligand atoms, which were created at chosen LBSs as centroids, was performed. The resulting $\mathrm{U}_{\mathrm{DOCK}}$ was calculated. An $\alpha$-D-mannose was properly added to $\operatorname{Tr}^{130}$ of human HYAL1 to be $C$-mannosylated within MOE, and then the energy of $C$-mannosylated human HYAL1 was minimized. Protein docking between $C$-mannosylated human HYAL1 and dummy ligand atoms was performed. The resulting $\mathrm{U}_{\text {DOCK }}$ was calculated. 
A
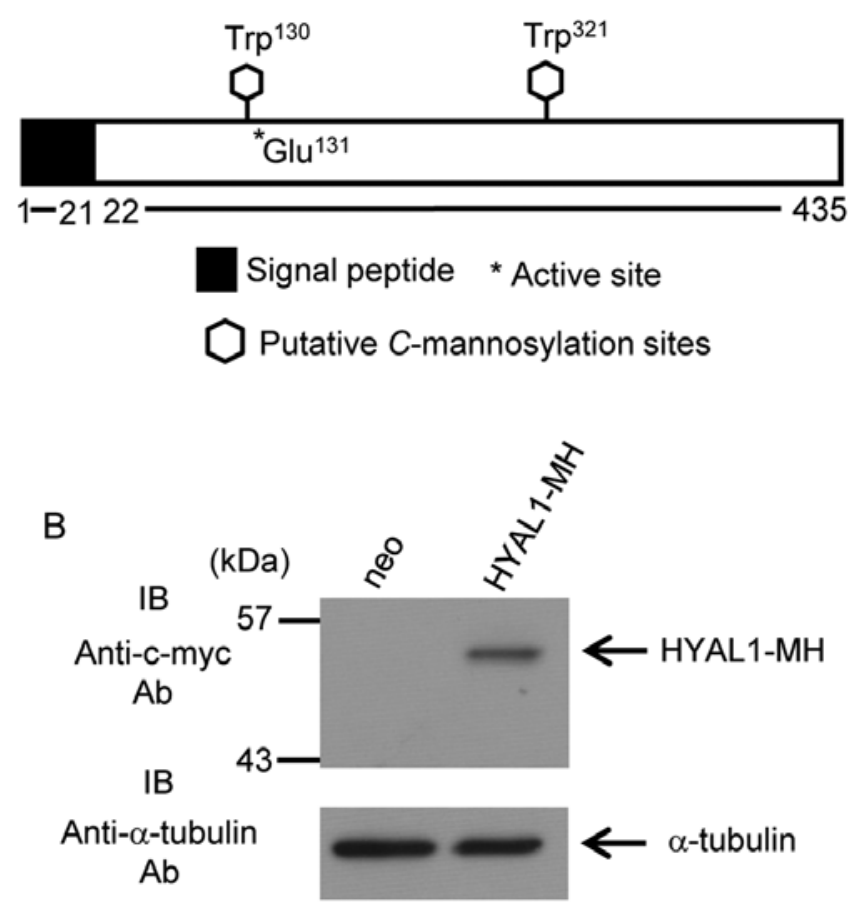

Figure 1.Establishment of HYAL1-overexpressing cell line. (A) Schematic diagram of human HYAL1. The locations of two putative $C$-mannosylation sites $\left(\operatorname{Tr} \mathrm{p}^{130}\right.$ and $\left.\operatorname{Tr}^{321}\right)$ are indicated by hexagonal shapes. A black box denotes the signal peptide. The active site of HYAL1 $\left(\mathrm{Glu}^{131}\right)$ is indicated by an asterisk (*). (B) Establishment of HYAL1-overexpressing cell line. Exponentially growing HT1080-neo and HT1080-HYAL1-MH cells were lysed, and each cell lysate was electrophoresed and immunoblotted with the indicated antibodies.

\section{Results}

Secreted HYAL1 has enzymatic activity, but is not $C$-mannosylated. Human HYAL1 contains two predicted $C$-mannosylation consensus sequences, $\operatorname{Tr} \mathrm{p}^{130}$ and $\operatorname{Tr} \mathrm{p}^{321}$. The active site of HYAL1 $\left(\mathrm{Glu}^{131}\right)$ is located next to $\operatorname{Trp}^{130}$ (Fig. 1A). To examine whether HYAL1 is $C$-mannosylated or not, we established HT1080 cells that stably expressed HYAL1 (Fig. 1B).

HYAL1 has a signal peptide at the N-terminus domain (Fig. 1A) and is predicted to secrete. We examined whether HYAL1 secretes or not and confirmed HYAL1 secretion (Fig. 2A). Furthermore, secreted HYAL1 possessed enzymatic activity (Fig. 2A; see alcian blue staining). We undertook MALDI-TOF MS analysis to determine whether secreted HYAL1 is actually $C$-mannosylated (30). To obtain recombinant HYAL1 protein, we purified HYAL1 from conditioned medium of HT1080-HYAL1-MH cells by using Ni-NTA agarose (data not shown). Purified HYAL1 was treated with trypsin, and the resulting mixture of the peptides was analyzed by MALDI-TOF MS (Fig. 2B and C). C-mannosylation, the attachment of one mannose to a tryptophan residue, should provoke an increase of $m / z$ 162. The peptides containing $\operatorname{Tr} p^{130}$ were observed at $m / z, 3,156.0$, but no peaks around at $m / z, 3,318$, which is the mass resulting from $C$-mannosylation of the peptide, were detected (Fig. 2B). In the same way, we performed MS analysis of the peptides containing $\operatorname{Trp}^{321}$, and the peak was observed
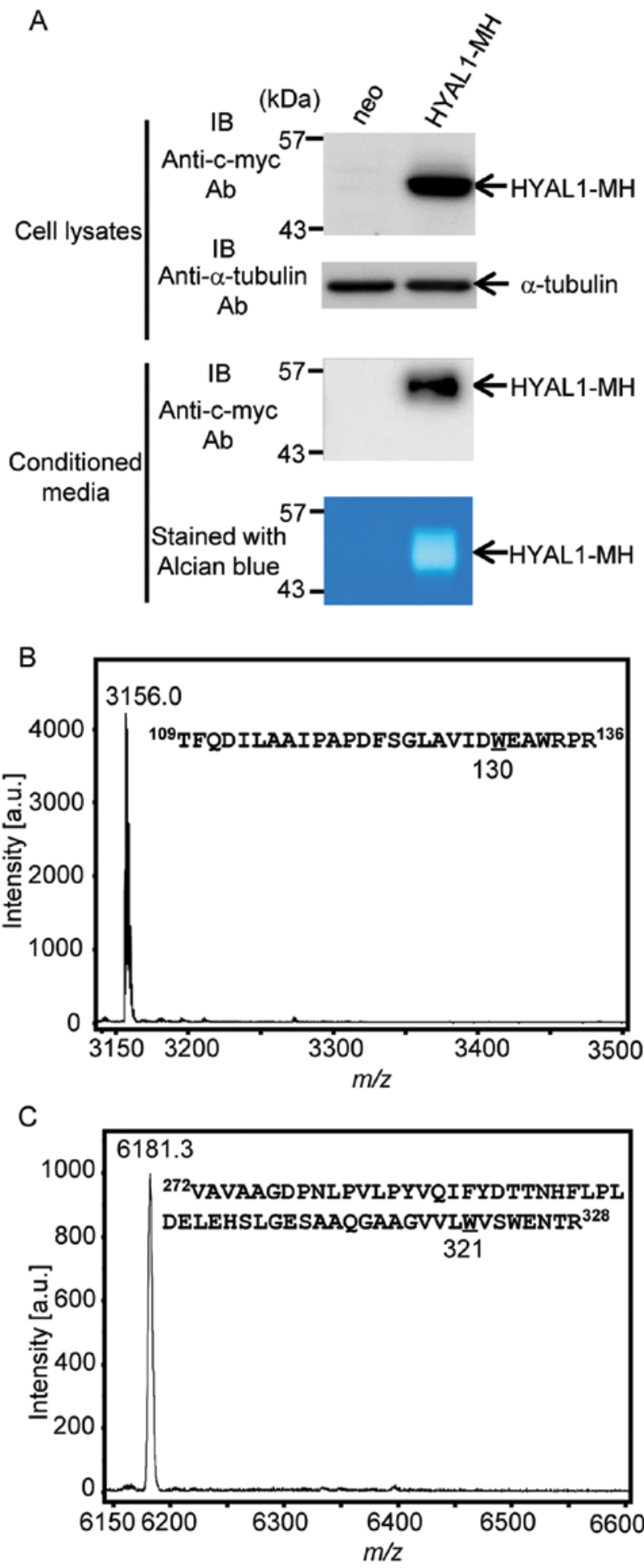

Figure 2. Secreted HYAL1 is not $C$-mannosylated. (A) HYAL1 was secreted into the conditioned medium. Exponentially growing HT1080-neo and HT1080-HYAL1-MH cells were cultured in serum-free DMEM for $24 \mathrm{~h}$, before the conditioned media were collected. The obtained samples from conditioned media were incubated with Ni-NTA agarose. Ni-NTA-bound HYAL1 was eluted with $500 \mathrm{mM}$ imidazole. Obtained samples were electrophoresed and immunoblotted with the indicated antibodies. For measurement of enzymatic activity, samples were electrophoresed on an SDSpolyacrylamide gel containing $0.2 \mathrm{mg} / \mathrm{ml} \mathrm{HA}$ at $4^{\circ} \mathrm{C}$. The gel was incubated at $37^{\circ} \mathrm{C}$ for $24 \mathrm{~h}$ after extraction of SDS and stained with alcian blue solution. (B and C) Representative MALDI-TOF MS data for peptides from the conditioned medium containing (B) $\operatorname{Trp}^{130}$ and (C) $\operatorname{Tr}^{321}$. Purified HYAL1 from the cell culture-conditioned medium was digested with trypsin, and the resulting peptides were analyzed by MALDI-TOF MS. Unmannosylated peptides containing $\operatorname{Trp}^{130}$ and $\operatorname{Trp}^{321}$ were observed at $m / z$ (B) 3,156.0 and (C) $6,181.3$, respectively. 

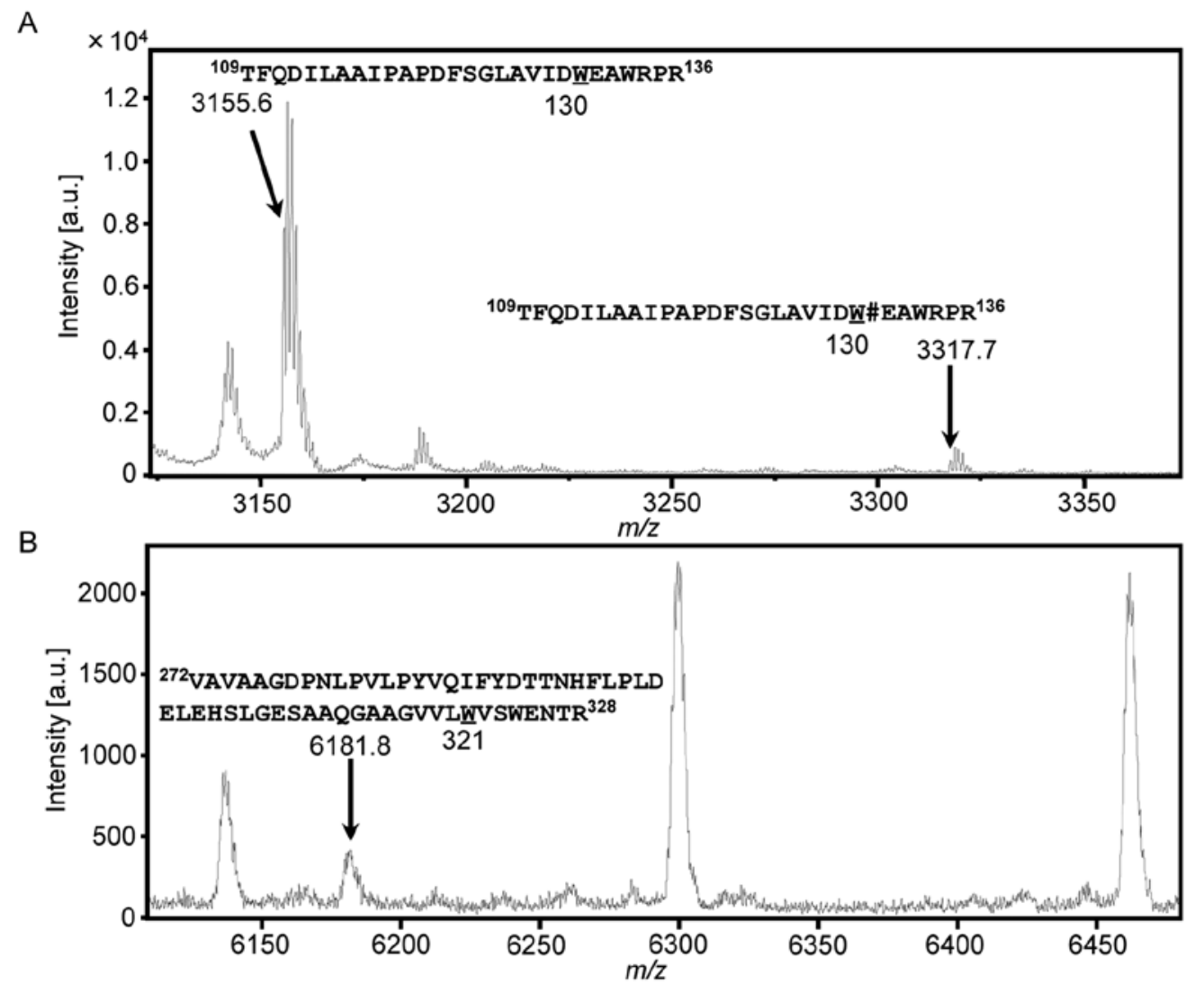

C

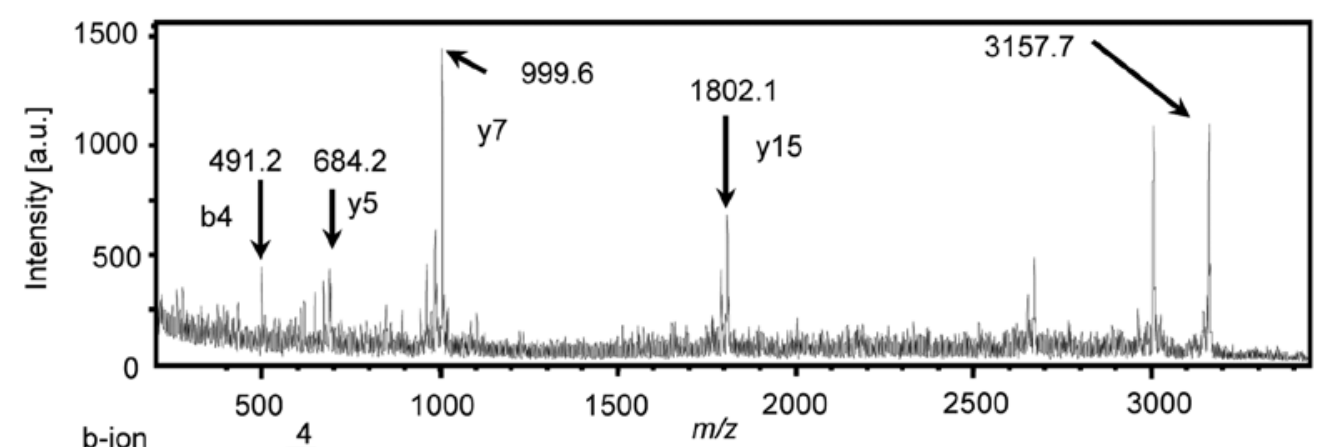

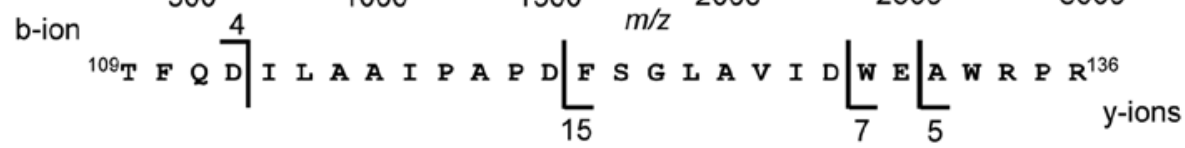

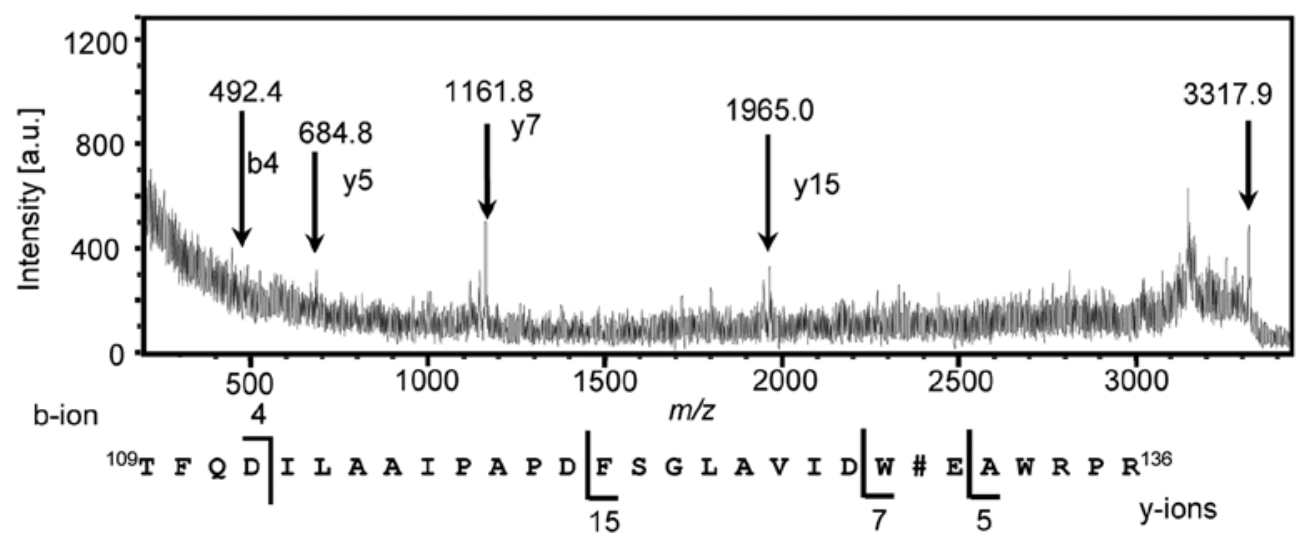

Figure 3. MALDI-TOF MS analysis reveals attachment of a mannose within intracellular HYAL1. (A) Representative MALDI-TOF MS data for the peptide from whole-cell lysates containing $\operatorname{Tr}^{130}$. Purified HYAL1 from whole-cell lysates was digested with trypsin, and the resulting peptides were analyzed by MALDI-TOF MS. The peaks of mannosylated and unmannosylated peptides were detected at $m / z, 3,317.7$ and 3,155.6, respectively. $C$-mannosyltryptophan is indicated by W\#. (B) Representative MALDI-TOF MS data for the peptide from whole-cell lysates containing Trp ${ }^{321}$. Purified HYAL1 from whole-cell lysates was digested with trypsin, and the resulting peptides were analyzed by MALDI-TOF MS. Only the peak of unmannosylated peptides was detected at $m / z$ 6,181.8. (C and D) Representative MALDI-TOF MS/MS data for the peptide containing $\operatorname{Tr}^{130}$. (C) Unmannosylated and (D) mannosylated peptides were analyzed by MALDI-TOF MS/MS. Observed peaks of these fragments are indicated as b-ion and y-ions. $C$-mannosylated Trp residue is indicated by W\#. 


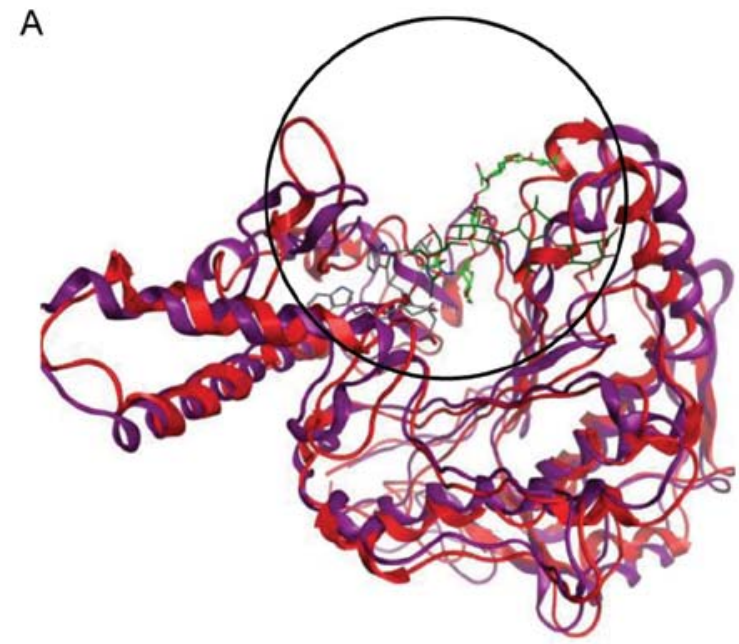

B

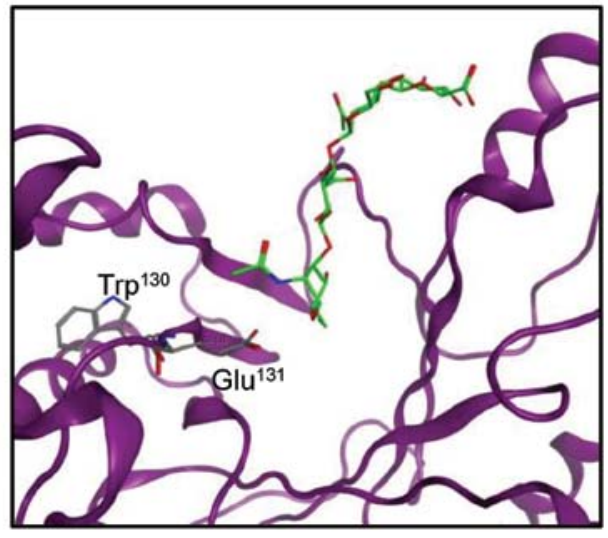

C

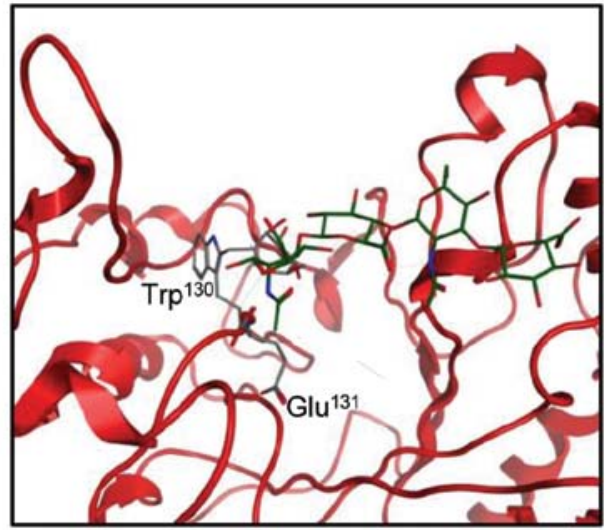

Figure 4. HYAL1 conformation is highly affected by $C$-mannosylation. (A) Structures of mannosylated HYAL1-HA and unmannosylated HYAL1-HA. Using the structures of Apis melliflora hyaluronidase-HA complex (PDB, 1FCV) and human HYAL1 (PDB, 2PE4), alignment of human hyaluronidase-HA complex was performed. Protein docking was performed between mannosylated HYAL1 and HA or unmannosylated HYAL1 and HA by MOE-ASE-Dock. The resulting free energy was also calculated. Mannosylated and unmannosylated HYAL1 are indicated with red and purple, respectively. (B and C) Close-ups of around the black circle in panel A. Unmannosylated HYAL1-HA and mannosylated HYAL1-HA complexes are indicated in panels B and C, respectively.

at $m / z 6,181.3$, which indicated that it was an unmannosylated peptide (Fig. 2C). However, the peak that was located at about $m / z, 6,343$, which is the mass resulting from $C$-mannosylation of the peptide, was not detected (Fig. 2C). These results revealed that secreted HYAL1 was not $C$-mannosylated, although secreted HYAL1 possessed enzymatic activity.

Determination of the mannose attachment residue within HYAL1 by using MALDI-TOF MS. Since HYAL1 is reported to lie mainly in lysosomes (31), we tried to examine whether intracellular HYAL1 is $C$-mannosylated. We purified HYAL1 proteins from whole-cell lysates of HT1080-HYAL1-MH cells (data not shown), and the obtained recombinant HYAL1 was treated with trypsin. The resulting mixture of peptides that were digested by trypsin was analyzed by MALDI-TOF MS (Fig. 3A and B). The peptides containing $\operatorname{Tr} \mathrm{p}^{130}$ were observed at $m / z$ 3,155.6, which were unmannosylated peptides (Fig. 3A). Moreover, a peak at $\mathrm{m} / z$ 3,317.7, which was an increase of $\mathrm{m} / \mathrm{z}$ 162, was also observed (Fig. 3A). It suggested that the peptides containing $\operatorname{Tr} \mathrm{p}^{130}$ were $C$-mannosylated. We performed MS analysis on the peptides containing $\operatorname{Trp}^{321}$, and a peak was observed at only $m / z 6,181.8$, which was predicted to be an unmannosylated peptide (Fig. 3B). These results indicated that intracellular HYAL1 was $C$-mannosylated at only $\operatorname{Trp}^{130}$. We further analyzed the peptides containing $\operatorname{Tr}^{130}$ by MALDI-TOF MS/MS to confirm the mannose attachment residue (Fig. 3C and D). Unmannosylated peptides (Fig. 3C) and mannosylated peptides (Fig. 3D) were analyzed, and b4 and $y 5$ ions were observed at the same position. However, y7 and y15 ions were observed at the $\mathrm{m} / \mathrm{z}$ 162-increased positions in mannosylated peptides (Fig. 3D) compared with unmannosylated peptides (Fig. 3C). These results confirmed that $C$-mannosylation of HYAL1 occurred at $\operatorname{Tr}^{130}$ only in the cell lysate, not the secreted HYAL1, suggesting that $C$-mannosylation might suppress HYAL1 secretion.

HYAL1 conformation is highly affected by C-mannosylation. Since secreted HYAL1 showed enzymatic activity (Fig. 2A), $C$-mannosylation may not be essential for HYAL1 activity. However, since $C$-mannosylated $\operatorname{Trp}^{130}$ lies next to $\mathrm{Glu}^{131}$, the active site of HYAL1, we assumed that $C$-mannosylation might regulate HYAL1 enzymatic activity. In order to examine the effect of $C$-mannosylation on HYAL1 enzymatic activity, we employed MOE, a computer simulation software that could calculate structural changes or free energies of interacting proteins. The crystal structure of human hyaluronidase-HA has not been reported, so we used the Protein Data Bank sequences for Apis melliflora hyaluronidase-HA complex (PDB: 1FCV) and human HYAL1 (PDB: 2PE4) in reference to a previous report (27) and performed protein alignment by the MOE tool to construct the human HYAL1-HA complex. The position of the active pocket of the constructed model 
accorded with 1FCV. We performed ASE-Dock between human HYAL1 and dummy atoms in the possible active pocket. As expected, HA was stably located at the active pocket, and the active site $\mathrm{Glu}^{131}$ nearly faced the HA cleavage site (Fig. 4A and B; docking energy, $-38.6 \mathrm{kcal} / \mathrm{mol}$ ). We next calculated the effect of $C$-mannosylation of HYAL1 on conformation or free energy. The docking simulation revealed that $C$-mannosylation changed the conformation, especially the nearby active pocket of HYAL1 (Fig. 4A and C). Moreover, the active site $\mathrm{Glu}^{131}$ faced in the opposite direction toward its substrate, HA (Fig. 4A and C; docking energy, $-2.45 \mathrm{kcal} / \mathrm{mol}$ ). These results suggest that $C$-mannosylation may cause the instability of HYAL1 conformation, resulting in suppression of HYAL1 enzymatic activity.

\section{Discussion}

In this report, we demonstrated that intracellular HYAL1 was $C$-mannosylated at $\operatorname{Trp}^{130}$ (Fig. 3), which has possible roles for secretion and enzymatic activity (Figs. 2 and 4). Some $C$-mannosylated proteins are found among TSR-1 superfamily proteins, such as thrombospondin, mindin, F-spondin and ADAMTS-like 1/punctin-1 (8); however, HYAL1 does not belong to the TSR-1 superfamily. This is the first report revealing the presence of $C$-mannosylation among the HYAL family. Although some reports demonstrated that $C$-mannosylation regulates secretion, other functions of $C$-mannosylation were scarcely reported; however, we suggest that $C$-mannosylation of HYAL1 might attenuate its enzymatic activity. Attachment of $\alpha$-D-mannose to the tryptophan residue by $C$-mannosylation changes protein polarity, because the polar D-mannose is attached to the non-polar tryptophan. Moreover, conformational change is also expected. These changes are predicted to affect some sorts of functions on $C$-mannosylated proteins, not only secretion, but also protein stability and/or enzymatic activity. Therefore, we anticipated that HYAL1 functions, such as secretion and enzymatic activity, were regulated by $C$-mannosylation. Surprisingly, however, secreted HYAL1 was not $C$-mannosylated at all (Fig. 2), although intracellular HYAL1 was partially modified (Fig. 3). These results suggest that $C$-mannosylation of HYAL1 negatively regulates the secretion of HYAL1 and that there is an unidentified enzyme that detaches mannose from $C$-mannosylated HYAL1.

MOE has been used widely in research for many purposes, such as homology modeling or docking simulation $(32,33)$. In this research, MOE demonstrated that $C$-mannosylation highly changed HYAL1 conformation and induced protein instability, so that we presumed that $C$-mannosylation had roles in enzymatic activity. By $C$-mannosylation of HYAL1, the active site Glu ${ }^{131}$ faced in the opposite direction toward $\mathrm{HA}$, and $\mathrm{Glu}^{131}$ did not face the cleavage position of HA. Moreover, $C$-mannosylated HYAL1 could no longer recognize $\mathrm{HA}$ as a ligand, according to Site Finder, which can search for possible active pockets of proteins (data not shown). Therefore, $C$-mannosylation disables HYAL1 to degrade HA. Although mannose is very small, the attachment of mannose to a $\operatorname{Tr}^{130}$ residue next to the active site $\mathrm{Glu}^{131}$ caused profound conformational changes. Furthermore, a previous report demonstrated that HYAL1 enzymatic activity was influenced by site-directed mutagenesis for various sites, such as the active site, disulfide bond sites or some $N$-glycosylation sites (27). This result means that proper conformation is required for its enzymatic activity and supports our prediction that $C$-mannosylation, which causes conformational change, is also important for its functions.

According to MOE, secretion and enzymatic activity of HYAL1 is predicted to be inhibited by $C$-mannosylation, therefore, we have established $C$-mannosylation-defective W130A mutant HYAL1 expressing cell line to evaluate the effects of $C$-mannosylation for HYAL1 functions. It is predicted that secretion and enzymatic activity of W130A mutant HYAL1 will be increased compared with wild-type HYAL1 although the ratio of $C$-mannosylated HYAL1 is small. However, secretion of W130A mutant HYAL1 was decreased compared with wild-type HYAL1 (data not shown). Moreover, we evaluated the enzymatic activity of purified secreted wild-type and W130A mutant HYAL1 purified from conditioned media, which were both unmannosylated. As a result, enzymatic activity of W130A mutant HYAL1 was also decreased compared with wild-type HYAL1 (data not shown). These results were inconsistent with our prediction, and we had concluded that these effects were not because of $C$-mannosylation but substitution from Trp to Ala. Therefore, in this report, we did not evaluate the effect of $C$-mannosylation by using $C$-mannosylationdefective mutant HYAL1.

Collectively, we demonstrated that HYAL1 was $C$-mannosylated at $\operatorname{Trp}^{130}$, and suggest the possible roles of $C$-mannosylation for secretion and enzymatic activity of HYAL1. Since HYAL1 is known to correlate with tumor malignancy, $C$-mannosylation of HYAL1 can be target for cancer therapeutics.

\section{Acknowledgements}

This study was supported in part by grants from the programs Grants-in-Aid for Scientific Research (B) (nos. 23310163 and 24310167) and for JSPS Fellows (254256). Y.N. is a Research Fellow of the Japan Society for the Promotion of Science.

\section{References}

1. Furmanek A and Hofsteenge J: Protein C-mannosylation: facts and questions. Acta Biochim Pol 47: 781-789, 2000.

2. Doucey MA, Hess D, Cacan R and Hofsteenge J: Protein C-mannosylation is enzyme-catalysed and uses dolichylphosphate-mannose as a precursor. Mol Biol Cell 9: 291-300, 1998.

3. Krieg J, Hartmann S, Vicentini A, Gläsner W, Hess D and Hofsteenge J: Recognition signal for C-mannosylation of Trp-7 in RNase 2 consists of sequence Trp-x-x-Trp. Mol Biol Cell 9: 301-309, 1998.

4. Julenius K: NetCGlyc 1.0: prediction of mammalian C-mannosylation sites. Glycobiology 17: 868-876, 2007.

5. Hofsteenge J, Müller DR, de Beer T, Löffler A, Richter WJ and Vliegenthart JF: New type of linkage between a carbohydrate and a protein: $\mathrm{C}$-glycosylation of a specific tryptophan residue in human RNase Us. Biochemistry 33: 13524-13530, 1994.

6. Gonzalez de Peredo A, Klein D, Macek B, Hess D, Peter-Katalinic J and Hofsteenge J: C-mannosylation and o-fucosylation of thrombospondin type 1 repeats. Mol Cell Proteomics 1: 11-18, 2002.

7. Hofsteenge J, Huwiler KG, Macek B, Hess D, Lawler J, Mosher DF and Peter-Katalinic J: C-mannosylation and O-fucosylation of the thrombospondin type 1 module. J Biol Chem 276: 6485-6498, 2001 
8. Wang LW, Leonhard-Melief C, Haltiwanger RS and Apte SS Post-translational modification of thrombospondin type-1 repeats in ADAMTS-like 1/punctin-1 by C-mannosylation of tryptophan. J Biol Chem 284: 30004-30015, 2009.

9. Hamming OJ, Kang L, Svensson A, Karlsen JL, Rahbek-Nielsen H, Paludan SR, Hjorth SA, Bondensgaard K and Hartmann R: Crystal structure of interleukin-21 receptor (IL-21R) bound to IL-21 reveals that sugar chain interacting with WSXWS motif is integral part of IL-21R. J Biol Chem 287: 9454-9460, 2012.

10. Buettner FF, Ashikov A, Tiemann B, Lehle L and Bakker $\mathrm{H}$ C. elegans DPY-19 is a C-mannosyltransferase glycosylating thrombospondin repeats. Mol Cell 50: 295-302, 2013.

11. Ihara Y, Manabe S, Ikezaki M, Inai Y, Matsui I-SL, Ohta Y, Muroi E and Ito Y: C-mannosylated peptides derived from the thrombospondin type 1 repeat interact with Hsc70 to modulate its signaling in RAW264.7 cells. Glycobiology 20: 1298-1310, 2010.

12. Muroi E, Manabe S, Ikezaki M, Urata Y, Sato S, Kondo T, Ito Y and Ihara Y: C-mannosylated peptides derived from the thrombospondin type 1 repeat enhance lipopolysaccharide-induced signaling in macrophage-like RAW264.7 cells. Glycobiology 17: 1015-1028, 2007.

13. Overall CM and Lopez-Otin C: Strategies for MMP inhibition in cancer: innovations for the post-trial era. Nat Rev Cancer 2: 657-672, 2002

14. Simizu S, Ishida K, Wierzba MK and Osada H: Secretion of heparanase protein is regulated by glycosylation in human tumor cell lines. J Biol Chem 279: 2697-2703, 2004.

15. Simizu $S$ and Niwa Y: Practical molecular targets for suppression of metastasis. For Immunopathol Dis Therap 4: 43-51, 2013.

16. Chao KL, Muthukumar L and Herzberg O: Structure of human hyaluronidase-1, a hyaluronan hydrolyzing enzyme involved in tumor growth and angiogenesis. Biochemistry 46: 6911-6920, 2007.

17. Tan JX, Wang XY, Su XL, Li HY, Shi Y, Wang L and Ren GS: Upregulation of HYAL1 expression in breast cancer promoted tumor cell proliferation, migration, invasion and angiogenesis. PLoS One 6: e22836, 2011.

18. Tan JX, Wang XY, Li HY, Su XL, Wang L, Ran L, Zheng K and Ren GS: HYAL1 overexpression is correlated with the malignant behavior of human breast cancer. Int J Cancer 128: 1303-1315, 2011.

19. Imundo L, Leduc CA, Guha S, Brown M, Perino G, Gushulak L, Triggs-Raine B and Chung WK: A complete deficiency of hyaluronoglucosaminidase 1 (HYAL1) presenting as familial juvenile idiopathic arthritis. J Inherit Metab Dis 34: 1013-1022, 2011.

20. Kuroda M, Funasaki S, Saitoh T, Sasazawa Y, Nishiyama S, Umezawa K and Simizu S: Determination of topological structure of ARL6ip1 in cells: identification of the essential binding region of ARL6ip1 for conophylline. FEBS Lett 587: $3656-3660,2013$.
21. Yasukagawa T, Niwa Y, Simizu S and Umezawa K: Suppression of cellular invasion by glybenclamide through inhibited secretion of platelet-derived growth factor in ovarian clear cell carcinoma ES-2 cells. FEBS Lett 586: 1504-1509, 2012.

22. Miyazaki I, Simizu S, Okumura H, Takagi S and Osada H: A small-molecule inhibitor shows that pirin regulates migration of melanoma cells. Nat Chem Biol 6: 667-673, 2010.

23. Simizu S, Umezawa K, Takada $M$, Arber $N$ and Imoto $M$ : Induction of hydrogen peroxide production and Bax expression by caspase-3(-like) proteases in tyrosine kinase inhibitorinduced apoptosis in human small cell lung carcinoma cells. Exp Cell Res 238: 197-203, 1998.

24. Simizu S, Suzuki T, Muroi M, Lai NS, Takagi S, Dohmae N and Osada $\mathrm{H}$ : Involvement of disulfide bond formation in the activation of heparanase. Cancer Res 67: 7841-7849, 2007.

25. Niwa Y, Suzuki T, Dohmae N, Umezawa K and Simizu S: Determination of cathepsin V activity and intracellular trafficking by N-glycosylation. FEBS Lett 586: 3601-3607, 2012.

26. Guntenhöner MW, Pogrel MA and Stern R: A substrate-gel assay for hyaluronidase activity. Matrix 12: 388-396, 1992.

27. Zhang L, Bharadwaj AG, Casper A, Barkley J, Barycki JJ and Simpson MA: Hyaluronidase activity of human Hyall requires active site acidic and tyrosine residues. J Biol Chem 284: 9433-9442, 2009

28. Yamaguchi H, Kidachi Y, Kamiie K, Noshita T and Uetsu H: Structural insight into the ligand-receptor interaction between glycyrrhetinic acid (GA) and the high-mobility group protei B1 (HMGB1)-DNA complex. Bioinformation 23: 1147-1153, 2012.

29. Susana DL, Lídia MG, Teresa AFC, Henrique FC, Rui M and Rita CG: Structure based virtual screening for discovery of novel human neutrophil elastase inhibitors. Med Chem Comm 3: 1299-1304, 2012.

30. Wilkins MR, Gasteiger E, Gooley AA, Herbert BR, Molloy MP, Binz PA, Ou K, Sanchez JC, Bairoch A, Williams KL and Hochstrasser DF: High-throughput mass spectrometric discovery of protein post-translational modifications. J Mol Biol 289: 645-657, 1999.

31. Frost GI, Csóka AB, Wong T, Stern R and Csóka TB: Purification, cloning, and expression of human plasma hyaluronidase. Biochem Biophys Res Commun 236: 10-15, 1997.

32. Azuma M, Kabe Y, Kuramori C, Kodo M, Yamaguchi Y and Handa H: Adenine nucleotide translocator transports haem precursors into mitochondria. PLoS One 3: e3070, 2008.

33. Fukiage C, Nakajima E, Ma H, Azuma M and Shearer TR: Characterization and regulation of lens-specific calpain Lp82. J Biol Chem 277: 20678-20685, 2002. 